Proceedings of the 9th Workshop on Quantum Chaos and Localisation Phenomena, May 24-26, 2019, Warsaw, Poland

\title{
Dual Approach to the Spectral Form Factor
}

\author{
N. HAHN*AND D. WALTNER \\ Fakultät für Physik, Universität Duisburg-Essen, Lotharstraße 1, 47048 Duisburg, Germany
}

\begin{abstract}
We study here the short-time behavior of the spectral form factor for chain-like many-body systems of arbitrary length $N$, an important tool to distinguish chaotic and integrable dynamics. We found in the past that while the long-time behavior of the spectral form factor is universal and follows predictions by Random Matrix Theory, it becomes highly system dependent for short times, even for large $N$ in the disorder-free case. Now, our aim is to study to which extend this observation persists if a nonzero disorder is considered.
\end{abstract}

DOI: 10.12693/APhysPolA.136.841

PACS/topics: spin chains, spectral form factor, disorder, perturbation theory

\section{Introduction}

The study of many-body systems is intimately connected with the need to deal with a large dimension of Hilbert space. Usually this dimension grows exponentially with the number of particles. A recently developed duality relation [1] overcomes this obstacle in the case of the short times, without the need for any further approximations for periodically kicked systems. In this context a dual operator, that is nonunitary in general, is derived. While the time evolution $\hat{U}_{N}$ evolves the system from a given time step to the next, the dual operator $\hat{W}_{T}$ evolves a given particle of the system for all $T$ time steps to the neighboring particle. The main gain achieved by introducing $\hat{W}_{T}$ is that spectral properties, i.e., the correlations of the eigenphases of the one-time-step evolution operator can be characterized analytically in the regime of small $T$ and arbitrary values of $N$. A quantity conviently used also for single-particle systems is the spectral form factor $[2,3]$

$$
K_{N}(T)=\frac{1}{2^{N}}\left|\operatorname{Tr} \hat{U}_{N}^{T}\right|^{2} .
$$

In this article we will study the kicked Ising Chain $[4,5]$ as an example of an interacting many-body system. In Ref. [5] consistency of the long time behavior of the spectral form factor was confirmed for the same system, with results from Random Matrix Theory (RMT) [6, 7]. By long times, we mean times beyond the Ehrenfest time $[8]$ that scales logarithmically with $\hbar$, and thus, becomes infinite in the semiclassical limit. The shorttime behavior in [5] was analyzed numerically. We described the short-time behavior of the spectral form factor analytically [1]. These studies were performed for a disorder-free system, i.e., the coupling between different spins and the on-site magnetic field to which the spins are exposed, were position independent. In contrast to the long-time behavior of the spectral form factor that is determined by universal RMT results depending

\footnotetext{
*corresponding author; e-mail: nico.hahn@stud.uni-due.de
}

only on $T$ and the Heisenberg time (the time conjugate to the mean level spacing via the Heisenberg uncertainty principal) the short-time behavior of the form factor is determined by the actual values of the system parameters (the couplings and the magnetic fields).

A further interesting property of the dual operator is related to its nonunitarity: while this operator is nonunitary in general, it can be unitary for a special choice of the couplings between the spins and the on-site magnetic field [1]. In this case, usually referred to as self-dual [9], the system has special properties: the spectral form factor in the presence of disorder in the $z$-component of the magnetic field is obtained in accordance with RMT in the thermodynamic limit $N \gg 1$ also for arbitrarily short times [9]. Furthermore, in Ref. [10] the system showed a linear increase of the entanglement entropy up to a saturation value. These two properties lead to the characterization of that system as maximally chaotic. Finally, this system shows special spatio-temporal structures of correlation functions [11]. In Ref. [12] we extended the analysis of Ref. [9] to parameter values close to but different from the self-dual situation, where we identified a transition to a localized behavior.

In this paper we want to follow a different route. Using the dual operator again we aim at analyzing the properties, especially the $N$-dependence of the spectral form factor, under the assumption of weak disorder in a numerical and analytical way. The major motivation for that is to understand to what extend the disorder helps to recover the Random Matrix Theory results also away from the self-dual situation.

The outline of this article is as follows: In Sect. 2 we introduce the Kicked Ising Chain model. In Sect. 3, we recapitulate the duality relation and show how it can be extended to disorder in the couplings between adjacent spins. Under the assumption that the couplings between the different spins of the chain vary only slightly, we obtain an analytical expression for $\operatorname{Tr} \hat{U}_{N}^{T}$ for $T=1$. This allows us to discuss the $N$-dependence of the spectral form factor in Sect. 4. In this context we consider our analytical approach developed in the Section before and also compute the form factor numerically. Finally, we conclude in Sect. 5. 


\section{Kicked Ising Chain}

Consider a chain of $N$ spin-1/2-particles with the following Hamiltonian

$$
\hat{H}(t)=\hat{H}_{I}+\hat{H}_{K} \sum_{\tau=-\infty}^{\infty} \delta(t-a \tau) .
$$

The interaction part $\hat{H}_{I}$ describes the next neighbour Ising interaction

$$
\hat{H}_{I}=\sum_{n=1}^{N} J_{n} \sigma_{n}^{z} \sigma_{n+1}^{z},
$$

and $\hat{H}_{K}$ describes the kicking of a magnetic field

$$
\hat{H}_{K}=\sum_{n=1}^{N} \boldsymbol{b}_{n} \cdot \boldsymbol{\sigma}_{n},
$$

where $\boldsymbol{\sigma}_{n}=\left(\sigma_{n}^{x}, \sigma_{n}^{y}, \sigma_{n}^{z}\right)$ are the spin operators (here Pauli matrices) for site $n$, and due to the boundary condition $\boldsymbol{\sigma}_{N+1}=\boldsymbol{\sigma}_{1}$. The coupling constant $J_{n}$ and the magnetic field $\boldsymbol{b}_{n}$ are, in general, site dependent, i.e., the system is only translationally invariant if $J_{n}=J$ as well as $\boldsymbol{b}_{n}=\boldsymbol{b}$ for all sites $n$. The magnetic field can be chosen as $\boldsymbol{b}_{n}=\left(b_{n} \sin \left(\varphi_{n}\right), 0, b_{n} \cos \left(\varphi_{n}\right)\right)$ without loss of generality. The Hamiltonian is periodic in time with period $a$ as $\boldsymbol{b}_{n}$ acts only at integer multiples of $a$. Thus, the Floquet operator (time evolution operator for one period) can be defined [2]

$$
\begin{aligned}
& \hat{U}=\mathcal{T} \exp \left(-\mathrm{i} \int_{0}^{a} \hat{H}(t) \mathrm{d} t\right)=\hat{U}_{I} \hat{U}_{K}, \\
& \hat{U}_{I}=\exp \left(-\mathrm{i} a \hat{H}_{I}\right), \quad \hat{U}_{K}=\exp \left(-\mathrm{i} \hat{H}_{K}\right) .
\end{aligned}
$$

with the time ordering operator $\mathcal{T}$. Here we can see that varying the time between kicks $a$ effctively leads to a rescaling of the coupling parameters $J_{n}$. The system becomes integrable for $\varphi=0$ as the Hamiltonian will be diagonal in the base of $\sigma^{z}$ and for $\varphi=\frac{\pi}{2}$. In the latter case the system can be mapped onto a chain of noninteracting spinless fermions via Jordan-Wigner transformation [13].

\section{Duality relation}

\subsection{Recapitualtion of the disorderfree case}

We are interested in the trace of the time evolution operator for $T$ time steps [1], namely:

$$
\operatorname{Tr} \hat{U}_{N}^{T} \text {. }
$$

A change of $a$ should not be confused with a change of $T$. Varying $a$ changes the time the system evolved, but not the number of kicks and actions of $\hat{U}_{I}$ involved. The latter is only changed by $T$. Henceforth we assume $a=1$. Due to the large dimension $2^{N} \times 2^{N}$ of the operator $\hat{U}$ we replace it by, in general, the non-unitary dual operator $\hat{W}_{T}$ of dimension $2^{T} \times 2^{T}$. This operator is constructed in such a way that its $N$-th power fullfils:

$$
\operatorname{Tr} \hat{U}_{N}^{T}=\operatorname{Tr} \hat{W}_{T}^{N} \text {. }
$$

This is because $\operatorname{Tr} \hat{U}_{N}^{T}$ resembles the partition function of a two dimensional Ising model on a $N \times T$ lattice with

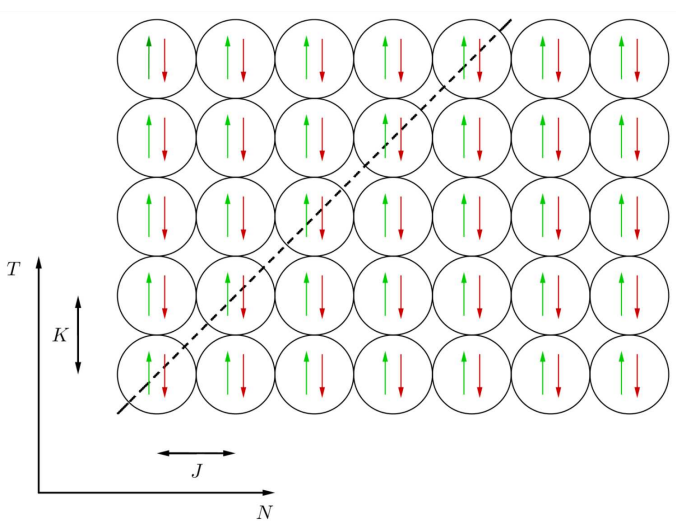

Fig. 1. The lattice of $N \times T$ dimension with coupling $J$ in $N$-direction and coupling $K$ in $T$-direction. The dashed line indicates the angle bisection.

the coupling $J$ of the original chain in the $N$-direction and the in general complex coupling $K$ (see below) in $T$-direction (c.f. Fig. 1). The partition function remains the same if we reflect the $N \times T$ lattice at its angle bisection. In this way we can think of a dual operator $\hat{W}_{T}$ that fullfils (7) and has the appropriate dimension. The complex coupling constant $K$ then leads to the nonunitarity of the dual operator.

The quantity $\hat{W}_{T}$ allows to characeterize especially the trace of the time evolution operator for an arbitrary number of particles for short times. It is given by a product of an Ising and a kick part

$$
\hat{W}_{T}=A \hat{U}_{I}(K) \hat{U}_{K}(\tilde{b}, \tilde{\varphi}) .
$$

The operators $\hat{U}_{I}$ and $\hat{U}_{K}$ have the same form as in Eq. (4), however the definition of parameters is different as in Eq. (4). Here, parameters are determined by

$$
\mathrm{e}^{-4 \mathrm{i} K}=1-\frac{1}{x^{2}} \quad \text { with } \quad x=\sin (b) \sin (\varphi),
$$

and

$$
\mathrm{e}^{-4 \mathrm{i} J}=1-\frac{1}{\tilde{x}^{2}} \quad \text { with } \quad \tilde{x}=\sin (\tilde{b}) \sin (\tilde{\varphi})
$$

with the additional condition

$$
\tan (b) \cos (\varphi)=\tan (\tilde{b}) \cos (\tilde{\varphi}) .
$$

The constant $A$ is given by

$$
A=\mathrm{e}^{T(\eta-\tilde{\eta})}
$$

with $\eta$ and $\tilde{\eta}$ determined by

$$
\mathrm{e}^{4 \eta}=x^{2}\left(x^{2}-1\right), \quad \mathrm{e}^{4 \tilde{\eta}}=\tilde{x}^{2}\left(\tilde{x}^{2}-1\right) .
$$

\subsection{Extension to disorder}

We will restrict our analysis here to site-independent magnetic fields $\boldsymbol{b}$, only the couplings $J_{n}$ are sitedependent. In the case of disorder, i.e., inhomogenous couplings $J_{n}$, the $N$-th power of $\hat{W}_{T}$ is replaced by the following product

$$
\hat{W}_{T}^{N}=B^{N} \hat{U}_{I}(K) \hat{U}_{K}\left(\tilde{b}_{N}, \tilde{\varphi}_{N}\right) \ldots \hat{U}_{I}(K) \hat{U}_{K}\left(\tilde{b}_{1}, \tilde{\varphi}_{1}\right) \text {. }
$$


The factors $\hat{U}_{I}(K)$ have the same form as in Eq. (8). The arguments $\tilde{b}_{N}$ and $\tilde{\varphi}_{N}$ are now given by

$$
\mathrm{e}^{-4 \mathrm{i} J_{n}}=1-\frac{1}{\tilde{x}_{n}^{2}} \quad \text { with } \quad \tilde{x}_{n}=\sin \left(\tilde{b}_{n}\right) \sin \left(\tilde{\varphi}_{n}\right) .
$$

The factor $B$ is given by

$$
B=\exp \left(T \sum_{n=1}^{N}\left(\eta-\tilde{\eta}_{n}\right) / N\right) .
$$

It is easy to check that the results given here turn into the ones without disorder for $J_{n}=J$.

\subsection{Approximation for $T=1$}

Under the condition that the magnetic field acts homogeneously on the chain and the coupling parameters vary only slightly, i.e.,

$$
J_{n}=J^{(0)}+J_{n}^{(1)},
$$

we can express the dual operator for $T=1$ by second order perturbation in $J_{n}^{(1)}$. The same can be done with the dual parameters:

$$
\begin{aligned}
& \tilde{b}_{n}=\tilde{b}^{(0)}+\tilde{b}_{n}^{(1)}, \\
& \tilde{\varphi}_{n}=\tilde{\varphi}^{(0)}+\tilde{\varphi}_{n}^{(1)}, \\
& \tilde{\eta}_{n}=\tilde{\eta}^{(0)}+\tilde{\eta}_{n}^{(1)} .
\end{aligned}
$$

Their values are determined by the $J_{n}^{(1)}$ via Eqs. (10) and (13). The prefactor from Eq. (16) evaluates to

$$
\begin{aligned}
B & =\mathrm{e}^{N\left(\eta-\tilde{\eta}^{(0)}\right)}\left[1-\sum_{n=1}^{N} \eta_{n}^{(1)}+\frac{1}{2}\left(\sum_{n=1}^{N} \eta_{n}^{(1)}\right)^{2}\right] \\
& +\mathcal{O}\left(\left(\eta_{n}^{(1)}\right)^{3}\right) .
\end{aligned}
$$

Note that for $T=1$ the Ising part will be a constant factor

$$
\hat{U}_{I}=\mathrm{e}^{-\mathrm{i} N K} .
$$

The kick part is given by

$$
\begin{aligned}
& \hat{U}_{K}=\prod_{n=1}^{N} \mathrm{e}^{-\mathrm{i} \tilde{\boldsymbol{b}}_{n} \cdot \boldsymbol{\sigma}_{1}}= \\
& \prod_{n=1}^{N} \cos \tilde{b}_{n}+\mathrm{i} \sin \tilde{b}_{n}\left(\sin \left(\tilde{\varphi}_{n}\right) \sigma_{1}^{x}+\cos \left(\tilde{\varphi}_{n}\right) \sigma_{1}^{z}\right),
\end{aligned}
$$

where the left hand order of the product must not be violated because the one particle operators $\mathrm{e}^{-\mathrm{i} \tilde{\boldsymbol{b}}_{n} \cdot \boldsymbol{\sigma}_{1}}$ do not commute in general. It can be expanded into second order by starting with the one particle operators

$$
\mathrm{e}^{-\mathrm{i} \tilde{\boldsymbol{b}}_{n} \cdot \boldsymbol{\sigma}_{1}}=\alpha+\beta_{n}+\frac{1}{2} \gamma_{n}+\mathcal{O}\left(\left(J_{n}^{(1)}\right)^{3}\right)
$$

with

$$
\begin{aligned}
& \alpha=\cos \tilde{b}^{(0)}-\mathrm{i} \sin \tilde{b}^{(0)} G\left(\tilde{\varphi}^{(0)}\right), \\
& \beta_{n}=-\mathrm{i} \tilde{\varphi}_{n}^{(1)} \sin \tilde{b}^{(0)} G\left(\tilde{\varphi}^{(0)}+\frac{\pi}{2}\right) \\
& \quad-\mathrm{i} \tilde{b}_{n}^{(1)} \cos \tilde{b}^{(0)} G\left(\tilde{\varphi}^{(0)}\right)-\tilde{b}_{n}^{(1)} \sin \tilde{b}^{(0)},
\end{aligned}
$$

$$
\begin{aligned}
& \gamma_{n}=-\left(\tilde{b}_{n}^{(1)}\right)^{2} \cos \tilde{b}^{(0)} \\
& \quad-2 \mathrm{i} \tilde{b}_{n}^{(1)} \tilde{\varphi}_{n}^{(1)} \cos \tilde{b}^{(0)} G\left(\tilde{\varphi}^{(0)}+\frac{\pi}{2}\right) \\
& \quad+\mathrm{i}\left[\left(\tilde{b}_{n}^{(1)}\right)^{2}+\left(\tilde{\varphi}_{n}^{1)}\right)^{2}\right] \sin \tilde{b}^{(0)} G\left(\tilde{\varphi}^{(0)}\right),
\end{aligned}
$$

where the abbreviation

$$
G\left(\tilde{\varphi}^{(0)}\right)=\sin \tilde{\varphi}^{(0)} \sigma_{1}^{x}+\cos \left(\tilde{\varphi}^{(0)}\right) \sigma_{1}^{z}
$$

is used. The whole product is then up to second order

$$
\begin{aligned}
& \prod_{n=1}^{N} \exp \left(-\mathrm{i} \boldsymbol{b}_{n} \cdot \boldsymbol{\sigma}_{1}\right)=\prod_{n=1}^{N} \alpha+\beta_{n}+\frac{1}{2} \gamma_{n}= \\
& \alpha^{N}+\sum_{n=1}^{N} \alpha^{N-n} \beta_{n} \alpha^{n-1}+\frac{1}{2} \sum_{n=1}^{N} \alpha^{N-n} \gamma_{n} \alpha^{n-1} \\
& \quad+\sum_{n=1}^{N-1} \sum_{k=n}^{N-1}\left(\alpha^{N-1-k} \beta_{k+1} \alpha^{k-n}\right) \beta_{n} \alpha^{n-1} \\
& \quad+\mathcal{O}\left(\left(J_{n}^{(1)}\right)^{3}\right)
\end{aligned}
$$

Going onwards we will omit the last term as in practice we want to choose a distribution of $J_{n}^{(1)}$ which is symmetric around zero. Therefore, the sum of terms $J_{n}^{(1)} J_{m}^{(1)}$ with $n \neq m$ vanishes (and the dual parameters are directly proportional to $\left.J_{n}^{(1)}\right)$. The desired trace of the Floquet operator is now given by

$$
\begin{gathered}
\operatorname{Tr} \hat{W}_{1}^{N}=B^{N} \mathrm{e}^{-\mathrm{i} N K}\left(\operatorname{Tr} \alpha^{N}+\sum_{n=1}^{N} \operatorname{Tr}\left(\alpha^{N-n} \beta_{n} \alpha^{n-1}\right)\right. \\
\left.+\frac{1}{2} \sum_{n=1}^{N} \operatorname{Tr} \alpha^{N-n} \gamma_{n} \alpha^{n-1}\right)+\mathcal{O}\left(\left(J_{n}^{(1)}\right)^{3}\right) .
\end{gathered}
$$

The eigenvalues of the unperturbed (i.e., free from disorder) one particle operators are

$$
\tilde{\lambda}_{ \pm}=\cos \tilde{b}^{(0)} \pm \mathrm{i} \sin \tilde{b}^{(0)} \text {. }
$$

The trace can be expressed in terms of them after diagonalization of $\alpha$

$$
\begin{gathered}
\operatorname{Tr} \hat{W}_{1}^{N}=B^{N} \mathrm{e}^{-\mathrm{i} N K}\left[\tilde{\lambda}_{+}^{N}+\tilde{\lambda}_{-}^{N}+\mathrm{i} \sum_{n=1}^{N} \tilde{b}_{n}^{(1)}\left(\tilde{\lambda}_{+}^{N}-\tilde{\lambda}_{-}^{N}\right)\right. \\
-\frac{1}{2} \sum_{n=1}^{N}\left(\tilde{b}_{n}^{(1)}\right)^{2}\left(\tilde{\lambda}_{+}^{N}+\tilde{\lambda}_{-}^{N}\right) \\
\left.+\mathrm{i}\left(\tilde{\varphi}_{n}^{(1)}\right)^{2} \sin \tilde{b}^{(0)}\left(\tilde{\lambda}_{+}^{N-1}-\tilde{\lambda}_{-}^{N-1}\right)\right] .
\end{gathered}
$$

\section{Spectral form factor}

The spectral form factor as the Fourier transform of the two-point correlation function contains information about the correlations of the eigenphases (eigenvalues of the Floquet operator) and can therefore act as tool to diagnose quantum chaos. It can be written as 


$$
K(T)=\frac{1}{2^{N}}\left|\operatorname{Tr} \hat{U}_{N}^{T}\right|^{2}=\frac{1}{2^{N}}\left|\operatorname{Tr} \hat{W}_{T}^{N}\right|^{2},
$$

where we already made use of the duality relation (7). We want to compare our results with the spectral form factor of the appropriate RMT ensemble, the circular orthogonal ensemble (COE):

$$
K_{\mathrm{COE}}(T)=\left\{\begin{array}{ll}
|T|(2-\ln (1+2|T|)) & \text { for }|T| \leq 1 \\
2-|T| \ln \left(\frac{2|T|+1}{2|T|-1}\right) & \text { for }|T|>1
\end{array} .\right.
$$

We want to analyze the behavior up to the Heisenberg time $T_{H}=2^{N}$. Therefore, a rescaling of the RMT form factor is necessary [5]

$$
K_{\mathrm{COE}}(T) \rightarrow K_{\mathrm{COE}}\left(\frac{T}{2^{N}}\right) .
$$

For the disorder average we will choose our set of $J_{n}^{(1)}$ according to a uniform distribution in $[-\Delta J, \Delta J]$ with $\Delta J$ being the disorder strength. First we will look at a small disorder where our approximation (28) is valid.

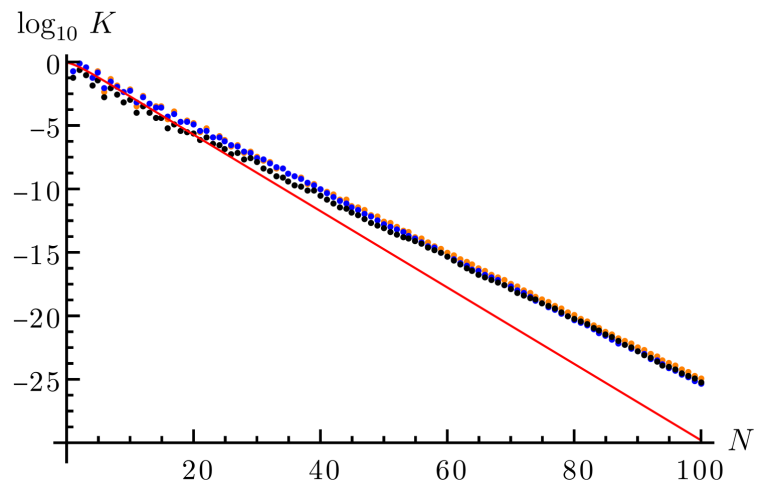

Fig. 2. Logarithmic plot of the spectral form factor as a function of $N$ within the chaotic regime $J^{(0)}=0.7$, $b=0.9 \sqrt{2}, \varphi=\frac{\pi}{4}$. The disordered case (blue - numeric, black - analytic approximation) with the disordered free case (orange) and the RMT result (red) for $\Delta J=0.2$.

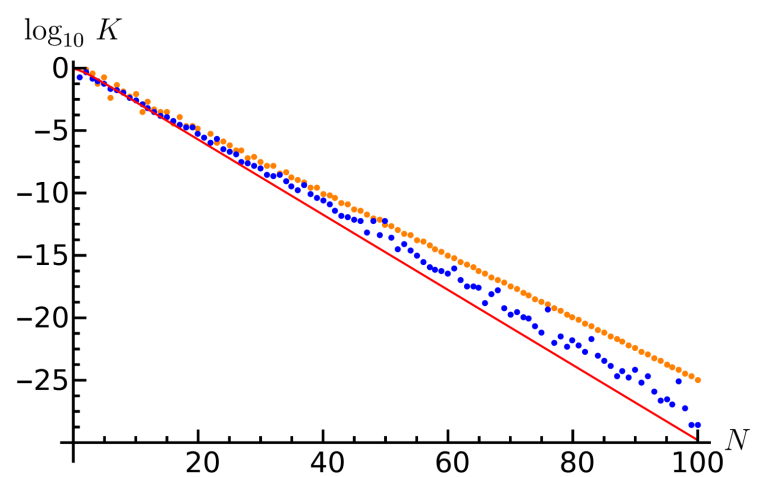

Fig. 3. Logarithmic plot of the spectral form factor as a function of $N$ within the chaotic regime $J^{(0)}=0.7$, $b=0.9 \sqrt{2}, \varphi=\frac{\pi}{4}$. The disordered case (blue), the disordered free case (orange) and the RMT result (red) for $\Delta J=1$.
In Fig. 2 we compare the RMT result with the disorderfree case and the disordered case in the weak disorder regime, where our perturbative approach is valid. We compare our analytical result with the one obtained by an exact numerical computation of the spectral form factor based on Eq. (14).

For larger $N$ we can see a good agreement between the numerical results and the approximation. For smaller particle numbers the fuctuations of the $J_{n}^{(1)}$ cause some deviations. The disordered case lies closely to the disorderfree case.

For larger disorder strength $\Delta J$ that lies beyond our analytical treatment, we can observe the effect that the disordered spectral form factor approaches the RMT results, c.f. Fig. 3.

\section{Conclusion}

We considered here the kicked Ising chain model [4], a prototype of an interacting many-body system, where we included disorder in the coupling between the adjacent spins, i.e., site-dependent couplings between the spins. Such a system is the more interesting as it combines interactions and disorder, and thus it yields the basis for the phenomenon of many-body localization, attracting huge interest in the recent years [14-17]. For such a system we analyzed the spectral form factor - a well established measure of quantum chaos $[2,3]$, i.e., a measure that yields a unique trace of classically integrable or chaotic dynamics in the discrete quantum spectrum. Therefore, we first extended the duality relation introduced in [1] for a disorder-free system to disorder in the couplings $J_{n}$. This allowed us to derive in the case of weak disorder an analytical expression for the trace of the dual operator, and thereby for the spectral form factor for one time step. In order to check our results the comparison with independent numerical computations was done and a very reasonable agreement was found. Furthermore we contrast our results with the predictions by RMT and observed that an increasing amount of disorder leads to a form factor approaching the one expected from RMT even for that short times. We see our approach as complementary to the ones in Refs. [9] and [12] that study the same quantity in the self-dual situation and in its vicinity, respectively.

Our analytical approach is restricted to $T=1$. It would be very interesting to extend it to larger times as well. We hope to confirm the increasing agreement in dependence of time with the Random Matrix Theory results, which we observed numerically in that way. Another research line would be to consider different quantities considering again the approximation of weak disorder. Examples are the entanglement entropy or spinspin correlation functions. Based on Refs. [10, 11] that study the self-dual situation it can be already expected that they show an interesting behavior in the short-time regime of the order of the duration between a few kicks. 


\section{Acknowledgments}

We want to thank Maram Akila, Petr Braun, and Thomas Guhr for discussions.

\section{References}

[1] M. Akila, D.Waltner, B. Gutkin, T. Guhr, J. Phys. A 49, 375101 (2016).

[2] H.J. Stöckmann, Quantum chaos-an introduction, Cambridge University press, 2006.

[3] F. Haake, S. Gnutzmann, M. Kuś, Quantum Signatures of Chaos, Springer Nature, Switzerland, 2019.

[4] T. Prosen, Prog. Theor. Phys. Suppl. 139, 191 (2000); Phys. Rev. E 65, 036208 (2002); J. Phys. A 40, 7881 (2007).

[5] C. Pineda, T. Prosen, Phys. Rev. E 76, 061127 (2007).

[6] M.L. Metha, Random Matrices, Elsevier, 2004.

[7] T. Guhr, A. Müller-Groeling, H.A. Weidenmüller, Phys. Rep. 299, 189 (1998).

[8] B.V. Chirikov, F.M. Izrailev, D.L. Shepelyansky, Sov. Sci. Rev. Sect. C 2, 209 (1981).
[9] B. Bertini, P. Kos, T. Prosen, Phys. Rev. Lett. 121, 264101 (2018).

[10] B. Bertini, P. Kos, T. Prosen, Phys. Rev. X 9, 021033 (2019).

[11] B. Bertini, P. Kos, T. Prosen, Phys. Rev. Lett. 123, 210601 (2019).

[12] P. Braun, D. Waltner, M. Akila, B. Gutkin, T. Guhr, arXiv:1902.06265.

[13] E. Lieb, T. Schultz, D. Mattis, Ann. Phys. 16, 407 (1961)

[14] B.L. Altshuler, Y. Gefen, A. Kamenev, L.S. Levitov, Phys. Rev. Lett. 78, 2803 (1997).

[15] D.M. Basko, I.L. Aleiner, B.L. Altshuler, Ann. Phys. 321, 1126 (2006).

[16] M. Žnidarič, T. Prosen, P. Prelovšek, Phys. Rev. B 77, 064426 (2008).

[17] M. Schreiber, S.S. Hodgman, P. Bordia, H.P. Lüschen, M.H. Fischer, R. Vosk, E. Altman, U. Schneider, I. Bloch, Science 349, 842 (2015). 